\title{
Shear strength of a Zr-based metallic glass over a wide temperature range
}

\author{
Cen Chen ${ }^{\text {a }}$, Baoan Sun ${ }^{\text {b,c,*, Wei Hua Wang }}{ }^{\text {b,c,** }}$, Tzu Chiang Wang ${ }^{\text {a,d }}$ \\ ${ }^{a}$ State Key Laboratory of Nonlinear Mechanics, Institute of Mechanics, Chinese Academy of Sciences, Beijing, 100190, China \\ ${ }^{\mathrm{b}}$ Institute of Physics, Chinese Academy of Sciences, Beijing, 100190, China \\ ' Songshan Lake Materials Laboratory, Dongguan, Guangdong, 523808, China \\ d School of Engineering Sciences, University of Chinese Academy of Sciences, Beijing, 100049, China
}

\section{A R T I C L E I N F O}

\section{Keywords:}

Metallic glasses

Shear strength

Shear band

\begin{abstract}
A B S T R A C T
The shear strength plays a critical role in constructing the yield criterion and in understanding the plastic deformation mechanism of glasses as well as designing their mechanical performance in engineering applications. However, experimentally measuring the shear strength of glasses remains a challenging issue due to their plastic flow dependent of the pressure/normal stress. Here, through a carefully designed mechanical method, we can measure the shear strength of metallic glasses (MGs) over a wide temperature range. The obtained strength data verify the cooperative shear model and the Mohr-Coulomb yield criterion, which are commonly used to describe the plastic flow behaviors of MGs. The pure shear strength data also results in a precise determination of some key parameters for constructing yield criterion and constitutive deformation law in MGs.
\end{abstract}

\section{Introduction}

The plastic flow mechanism of metallic glasses (MGs) has arouse considerable interests from the perspective of both fundamental science and engineering applications [1,2]. With a long-range disordered structure, MGs are deformed in a totally different manner as compared to crystalline alloys. Generally, the plastic flow of MGs is inhomogeneous with plastic strain highly localized into nanoscale shear bands [3-5]. The shear bands are prone to become runaway due to work softening, leading to the brittle fracture of MGs. The poor ductility of MGs has become an obstacle for their wide potential applications [6-8]. The origin of shear band instability is closely related to the shear-induced dilation at some local atomic-scale sites. Some theoretical models have been proposed to account for the atomic-scale deformation process, including free volume model [9], shear transformation zone (STZ) model [10] and the cooperative shear model (CSM) [11,12], etc.

One important consequence for the shear-induce dilation is that the plastic flow of MGs shows a dependence on the pressure/normal stress. As a result, the experimentally measured yield strength of MGs shows a significant variation under different loading modes. For example, under uniaxial loading, the plastic yielding of most bulk MGs is not along the maximum resolved shear stress plane $\left(45^{\circ}\right.$ inclined to the loading axis) [13], and the tensile and compressive strength values display obvious asymmetry [14], indicating that the plastic flow of MGs is not solely determined by the deviatoric stress part. The yielding strength measured from the uniaxial tests in fact contains a pressure/normal stress portion, which is difficult to be distinguished from that contributed by deviatroic stress $[15,16]$. However, in developing the deformation law and yield criterion of MGs, many theoretical models often use the shear strength which reflect the intrinsic shear resistance and do not vary with the loading conditions $[11,17,18]$. Therefore, a knowledge on the precise value of shear strength is highly desirable, which is important in constructing the yielding criterion and understanding the deformation behavior of MGs.

So far, measuring the shear strength of MGs remains a challenge in terms of traditional mechanical methods, and lack of systematical investigations. The torsion test, which had been applied for metallic material by the thin-walled tubular sample $[19,20]$, is obviously unsuitable for MGs due to their size limitation. For MGs, the cylinder sample seems to be the only choice for the torsion test [21], while the shear stress distributes linearly along the radial direction of the sample, which is difficult to determine the shear strength. Some other methods conducted pure shear tests, such as the single edge notched flexure fracture test [22], yet were just suitable for investigating the fracture toughness due to the introduction of pre-cracks and the stress concentration in samples. In this work, we designed a unique antisymmetrical four-point bend

\footnotetext{
* Corresponding author. Institute of Physics, Chinese Academy of Sciences, Beijing, 100190, China.

** Corresponding author. Institute of Physics, Chinese Academy of Sciences, Beijing, 100190, China.

E-mail addresses: sunba@iphy.ac.cn (B. Sun),whw@iphy.ac.cn (W.H. Wang).
} 
(anti-FPB) shear test method to measuring the shear strength of MGs. By using the method, we obtain the shear strength of MGs over a wide temperature range. These strength values verify the CSM theory and the Mohr-Coulomb $(\mathrm{M}-\mathrm{C})$ yielding criterion. The shear strength data over a wide temperature are important for constructing the precise yielding criterion and constitutive deformation law of glassy solids.

\section{Experimental methods}

The anti-FPB shear test method is effective to measure the shear strength for macroscopic brittle material [23,24]. As shown in Fig. 1a, the four loading rods, which are embedded into the grooved blocks, are placed anti-symmetrically in the plane for a plate sample. The force is transmitted to the loading rods from the press head of the machine, which renders the anti-symmetrical four-point loading mode for the sample. The illustrations of bending moment and shear force in Fig. 1b reveal that the pure shear stress field is realized at the anti-symmetrical center since the bending moment is zero at this position. To get a uniform shear stress field, the samples are machined with two aligned $90^{\circ}$ V-shape notches at the anti-symmetrical center [25] (see Fig. 1a and b). The previous studies found that the introduction of two aligned $90^{\circ}$ V-notches at the zero-moment section in the antisymmetrically loaded beam renders the shear-stress distribution quite uniform as the sides of the notches are parallel to the normal stress directions at that point in the test sample (see Fig. 1a and b) [26]. The photoelastic analysis also confirms that the uniform shear stress field between the two notch tips can be obtained [27].

We also analyzed the shear stress distribution in the anti-FPB shear samples with V-notches angles by finite element method (FEM) in our early work [25]. In the FEM analysis, we adopted the linear elastic constitutive model to obtain the macroscopic shear stress field by considering the fact that most of the MGs display little macroscopic plasticity before fracture $[7,28]$. The planar four nodes element is adopted and the total number of elements for the whole model is 118667. The element sizes at the notch tips and between the two notches tips are about $0.001 \mathrm{~mm}$. The simulation results show that the stress concentration appears near the notch tips for the samples with notch angle larger or less than $90^{\circ}$ [25]. While, the shear stress distributes uniformly for the $90^{\circ} \mathrm{V}$-notch samples [25]. In Fig. 1c, the shear stress distribution contour of finite element analysis further confirms that a uniform shear stress field at the anti-symmetrical center can be achieved for the anti-FPB shear sample with $90^{\circ} \mathrm{V}$-notches [25], which is suitable for measuring the shear strength of an isotropic glassy solid.

Alloy ingots with the nominal composition $\mathrm{Zr}_{52.5} \mathrm{Cu}_{17.9} \mathrm{Ni}_{14.6} \mathrm{Al}_{10} \mathrm{Ti}_{5}$ (Vit 105) were produced by arc melting a mixture of pure metals (purity $\geq 99.5 \%$ ) in a Ti-gettered argon atmosphere. To ensure compositional homogeneity, each ingot was re-melted at least three times. Plate-shape bulk metallic glass (BMG) samples with a thickness of $2 \mathrm{~mm}$ and a length of about $60 \mathrm{~mm}$ were obtained by suction casting into a copper mould. The amorphous nature of the as-cast specimens was examined by x-ray diffraction (XRD, PANalytical X'Pert PRO) with Co $\mathrm{K} \alpha$ radiation and differential scanning calorimetry (DSC, PerkinElmer DSC7).

Plate samples for the mechanical tests were cut from the BMG as-cast plate by a diamond saw with water cooling, and then were carefully ground and polished. The geometric size of samples is $l=50 \mathrm{~mm}, h=6$ $\mathrm{mm}, \delta=2 \mathrm{~mm}$. The samples were machined with two aligned $90^{\circ} \mathrm{V}$ notches at the anti-symmetrical center and the notch depth is $2.0 \mathrm{~mm}$. The samples were tested over a wide temperature range from $220 \mathrm{~K}$ $\left(0.31 T_{g}\right)$ to $620 \mathrm{~K}\left(0.88 T_{g}\right)$ with $20-40 \mathrm{~K}$ interval using the Instron 3384 system. All pure shear tests were repeated for three times with the displacement rate of $0.2 \mathrm{~mm} / \mathrm{min}$ and the heat rate of $10 \mathrm{~K} / \mathrm{min}$. When the test temperature was reached, each sample was held for 15-20 min before testing and the environmental cabinet surround the test facility was effective to maintain the test temperature in the loading process. After fracture, the fracture morphologies of all samples were examined carefully using a scanning electron microscopy (SEM).

\section{Results and discussions}

Fig. 2 presents the force-displacement curves for the Zr-based MG from lower to higher temperatures and the photos of fracture samples. The force-displacement curves in Fig. 2a show that all samples fail in catastrophic fracture and the fracture takes place without obvious macroscopic plastic deformation. Then, the yield strength is determined by the crossover point from elastic to plastic portions with a very small offset of $0.05 \%$ [29]. As shown in Fig. 1b for the enlarged portion at yield and fracture of force-displacement curve at $300 \mathrm{~K}$, one can see that (a)

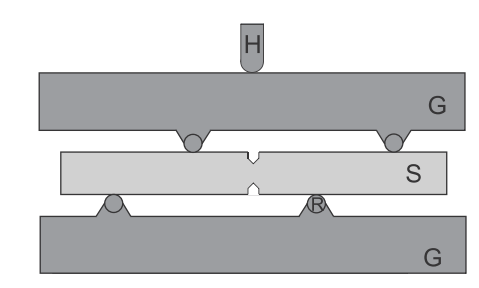

(c)

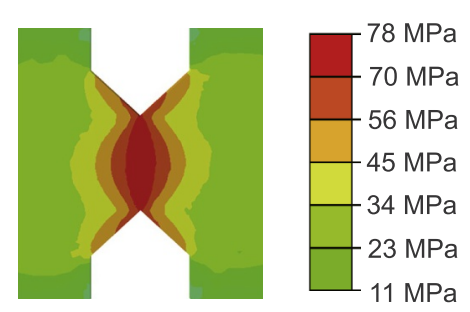

(b)
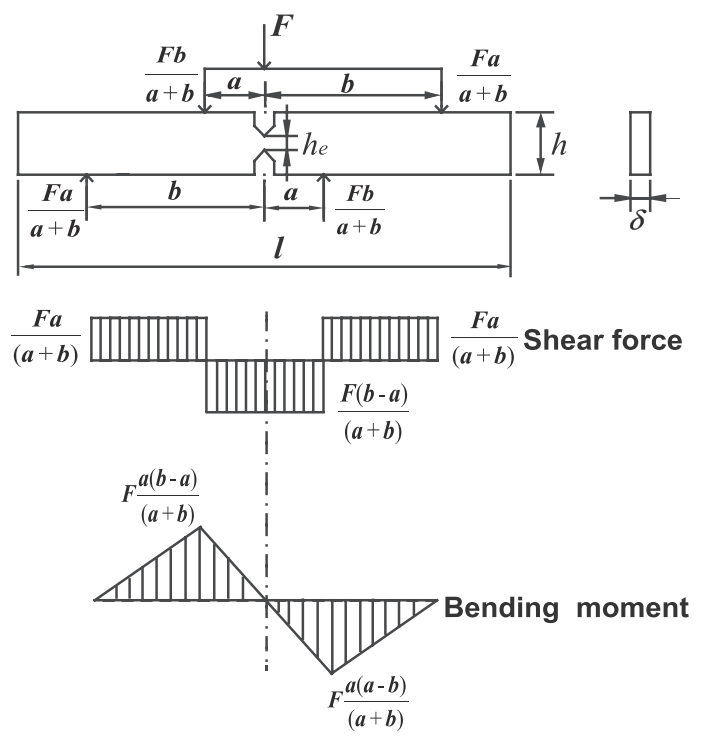

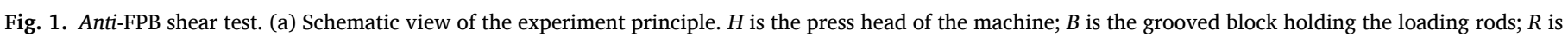

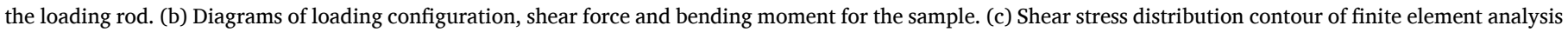
for the anti-FPB shear sample with two $90^{\circ}$ aligned V-notches. 
(a)

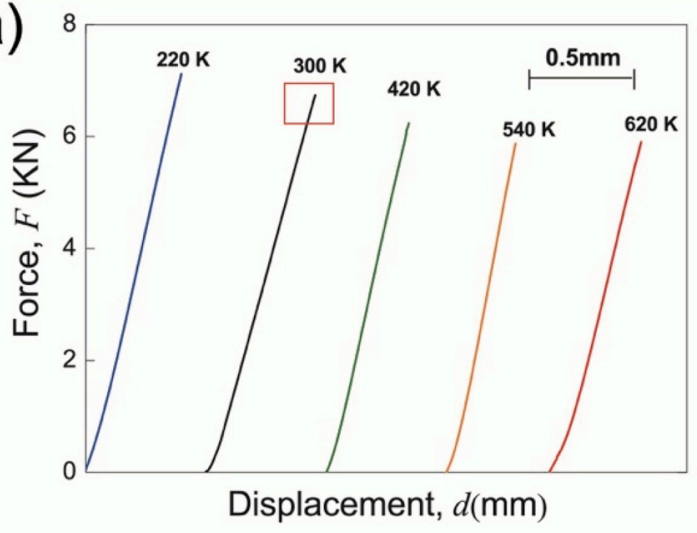

(c)

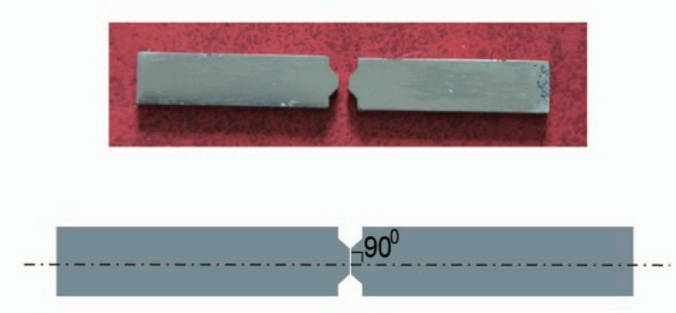

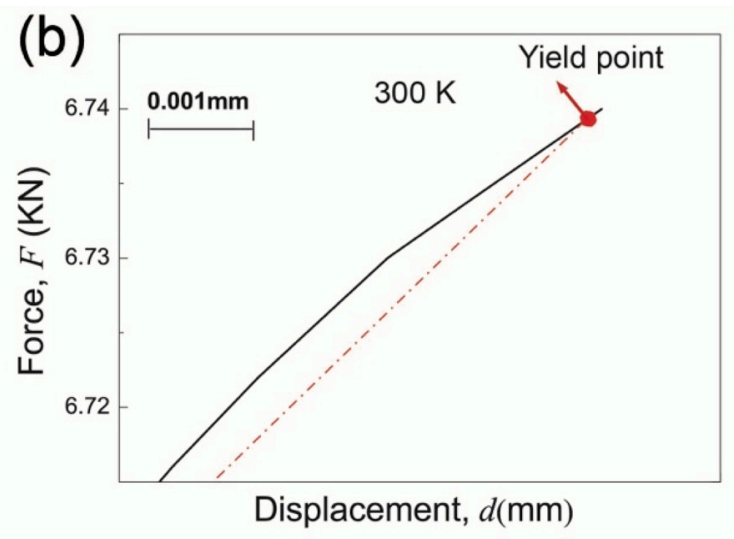

(d)

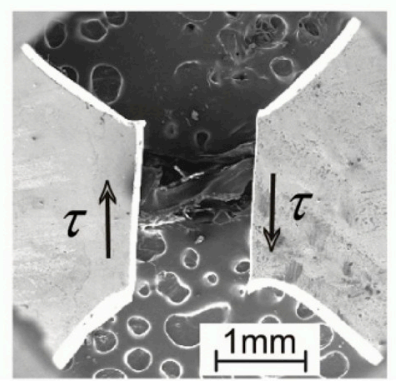

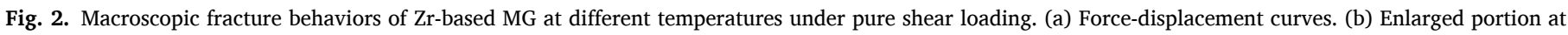
yield and fracture of force-displacement curve at $300 \mathrm{~K}$. (c) Photo for the fracture sample. (d) SEM for the fracture angle.

the fracture occurs as soon as the sample yields. The micrographs on the front sides of fracture samples in Fig. $2 \mathrm{~b}$ reveal that the crack propagates along the direction of the shear stress at different temperatures, which confirms that all samples underwent the pure shear. By comparing the SEM photos on the fracture samples (see Fig. 2c), we also find that the fracture of all samples occurs along the pure shear plane and the fracture angle does not change with the temperature.

The experimental shear strength can be obtained by,

$\tau_{0}=\frac{\mathrm{F}_{u}|b-a|}{\delta h_{e}(a+b)}$

where $F_{u}$ is the ultimate force at fracture. Since the yield and fracture occur almost simultaneous. The obtained values of shear strength in this work can represent both yield and fracture strength. The values for the shear strength $\tau_{0}$ at different temperatures are listed in Table 1 . As can be seen from Table 1, the shear strength decreases gradually with the increasing temperature, indicated that the reduced activation energy barrier for the plastic flow in MGs. For comparison, we also performed

Table 1

Measured value of shear strength $\left(\tau_{0}\right)$, half of compressive strength $\left(1 / 2 \sigma_{\mathrm{c}}\right)$ and friction coefficient $\alpha_{n}$ of $\mathrm{M}-\mathrm{C}$ yield criterion at different temperatures.

\begin{tabular}{lllll}
\hline$T(\mathrm{~K})$ & $T / T_{\mathrm{g}}$ & $\tau_{0}(\mathrm{GPa})$ & $1 / 2 \sigma_{\mathrm{c}}(\mathrm{GPa})$ & $\alpha_{n}$ \\
\hline 220 & 0.31 & 0.891 & - & - \\
260 & 0.37 & 0.942 & 0.843 & - \\
300 & 0.42 & 0.842 & 0.920 & 0.0886 \\
340 & 0.48 & 0.838 & 0.914 & 0.0867 \\
380 & 0.54 & 0.778 & 0.883 & 0.127 \\
420 & 0.60 & 0.780 & 0.873 & 0.113 \\
460 & 0.65 & 0.783 & 0.833 & 0.0609 \\
500 & 0.71 & 0.721 & 0.812 & 0.119 \\
540 & 0.76 & 0.724 & 0.780 & 0.0740 \\
570 & 0.80 & 0.691 & 0.731 & 0.0550 \\
600 & 0.85 & 0.789 & 0.617 & - \\
620 & 0.88 & 0.763 & 0.490 & - \\
\hline
\end{tabular}

compression tests on the MGs at the same temperature points and extracted the compressive strength from the stress-strain curve [30]. The half of the measured compressive strength $\left(1 / 2 \sigma_{\mathrm{c}}\right)$ is also listed in Table 1 and compared with the shear strength $\left(\tau_{0}\right)$. One can see that for the intermediate temperature range (from $300 \mathrm{~K}$ to $570 \mathrm{~K}$ ) $\tau_{0}$ is smaller than $1 / 2 \sigma_{\mathrm{c}}$. This is reasonable since the plastic shear of MGs is influenced by the pressure/normal stress. As shown in Fig. 3, the applied stress $\sigma$ for tensile and compressive samples of MGs can be resolved into two components [25]: the shear stress $\tau$ in the plane of flow, which makes the two parts of the sample slide over each other, and the normal stress $\sigma_{n}$, which is perpendicular to the plane of flow. Then, the resolved shear stress on the tensile fracture plane can be calculated by $\tau_{T}=$ $\sin \theta_{T} \cdot \cos \theta_{T} \cdot \sigma_{T}$, where $\sigma_{T}$ is tensile strength and $\theta_{T}$ is tensile fracture angle [31]. The resolved shear stress on the compressive fracture plane is $\tau_{C}=\sin \theta_{C} \cdot \cos \theta_{C} \cdot \sigma_{C}$, where $\sigma_{C}$ is compressive strength, and $\theta_{C}$ is compressive fracture angle [31]. Obviously, the tensile normal stress tries to pull the two parts of the sample apart and makes the sample easier to fracture at lower resolved shear stress, but the compressive normal stress extrudes the sample and makes the final fracture occur at higher resolved shear stress [32]. Then, we find that the shear strength

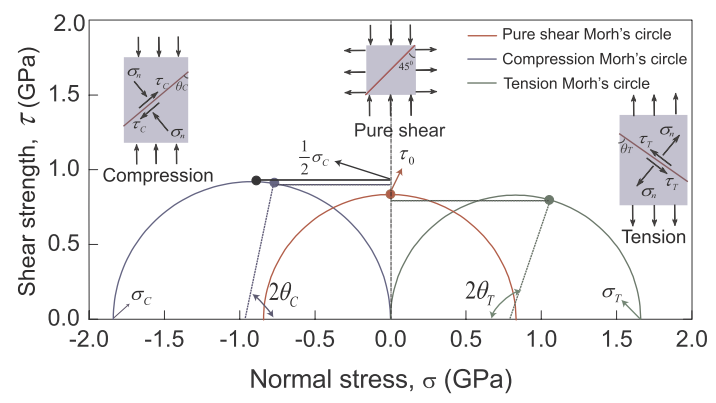

Fig. 3. Stress resolution on the tensile/compressive fracture surface by the Mohr's circle and comparison between the half of compressive strength $\left(1 / 2 \sigma_{c}\right)$ with the shear strength $\left(\tau_{0}\right)$. 
$\tau_{0}$ should satisfy that $\tau_{T}<\tau_{0}<\tau_{C}$. It is worth noting that when the temperature is lower than $260 \mathrm{~K}\left(0.37 T_{g}\right)$ or higher than $600 \mathrm{~K}\left(0.85 T_{g}\right)$, $\tau_{0}<\left(\sigma_{C} / 2\right)$ does not hold. This may be due to the transition of deformation mode as temperature is decreased or increased. In the intermediate temperature range, most MGs will fracture along a primary shear band or plane, which is closely related to the activation of STZs at the atomic scale. However, recent studies have showed that a transition from shear-dominated failure to dilation-dominated failure occurs when MGs at deformed at very low temperatures [33]. This transition is accompanied by the appearance of some flower-like vein patterns on the fracture surface at low temperatures, which is different from the dimple-like patterns as observed at room temperature. While at the high temperature (above $0.8 T_{g}$ ), a transition from the inhomogeneous deformation (shear banding) to homogeneous deformation also occurs, and this transition has been well documented in literatures [34]. Therefore, transition of deformation modes or mechanism at low or high temperatures may induce the failure of the rule that the shear strength is smaller than the half of compressive strength in MGs. Our speculation is also verified by the different fracture patterns in the shear tests observed at different temperatures, as can be seen in Fig. 4.

Fig. 5a displays the experimental data and theoretical calculation result of CSM for the shear strengths at different temperatures. The error bar represents the standard deviation of the measured data, which is less than $5 \%$ for each temperature. For the scaling law $\tau / G=\gamma_{0}-\gamma_{1}\left(T / T_{g}\right)^{2 / 3}$ of CSM, where $T$ represents temperature, $T_{g}$ is the glass transition temperature, $\tau$ is the shear strength at temperature $T, G$ is the shear modulus, the coefficients $\gamma_{0}=0.036 \pm 0.002$ and $\gamma_{1}=0.016 \pm 0.002$ are approximately universal constants. For Vit $105, \mathrm{G}=32.3 \mathrm{GPa}, T_{g}=708$ $\mathrm{K}$ and we set that $\gamma_{0}=0.033, \gamma_{1}=0.012$. The comparison between the experimental data and the CSM reveals that the shear strength is in line with the $\left(T / T_{g}\right)^{2 / 3}$ scaling law (see the black solid line and red circular symbols in Fig. 5a). And if we change the value of the coefficients $\gamma_{0}$ and $\gamma_{1}$, one can see that the CSM would agree well with the experimental results when $\gamma_{0}=0.033, \gamma_{1}=0.012$. Then, considering the error bar of experimental data, we calculate the upper and lower limits for the experimental data (see the red dash and dot lines in Fig. 5a). For the upper limit, $\gamma_{0}=0.0355, \gamma_{1}=0.012$; and for the lower limits, $\gamma_{0}=$ $0.0333, \gamma_{1}=0.014$.

The CSM had been proposed more than ten years and offers an important basis for understanding the plastic yield of MGs. While, the validation of CSM is inappropriate since that the shear strength is estimated by the compressive strength. Our experimental results indicate that the estimation of shear strength $\tau_{0}$ by the half of compressive strength is improper and the normal stress effect should not be ignored (see Fig. 3 and Table 1). Moreover, the CSM is carefully verified based on the experimental results in Fig. 5a, which shows that the $\left(T / T_{g}\right)^{2 / 3}$ scaling law in CSM can describe the plastic flow of MGs at finite temperature. While, the coefficients $\gamma_{0}$ and $\gamma_{1}$ in the CSM still need to adjust for more precise predictions. Therefore, our work offers a direct evidence for the validation of CSM and the construct of yielding criterion. And for the shear fracture, the normal stress effect is ruled out and the plastic flow of MGs only depends on the shear stress, which reveals the deformation law for the shear flow of MGs directly.

The data of shear strength at different temperatures are also helpful for constructing the precise plastic yield criterion in MGs. Many studies revealed that the pressure/normal stress plays an important role in the plastic yielding and flow of MGs. As a result, some yielding criterions incorporating the effect of hydrostatic element of stress tensor are often used to describe the plastic yielding of MGs. For example, the M - C yield criterion takes into account the effect of the normal component of stress on the shear plane, is written as:

$\tau_{y}=k_{y}-\alpha_{n} \sigma_{n}$

where $\tau_{y}$ is the yielding shear stress on the shearing plane, $\sigma_{n}$ is the normal stress on the shearing plane, $k_{y}$ is the shear strength or cohesive shear strength of the MG and $\alpha_{n}$ is a friction coefficient, which describes the normal stress dependence of the plastic shear and is important for understanding the nature of shear dilation. For the uniaxial compression or tension, the yielding shear stress and normal stress on a given shear plane can be easily calculated from the uniaxial yielding strength. Therefore, if the shear strength is known, the friction coefficient $\alpha_{n}$ can
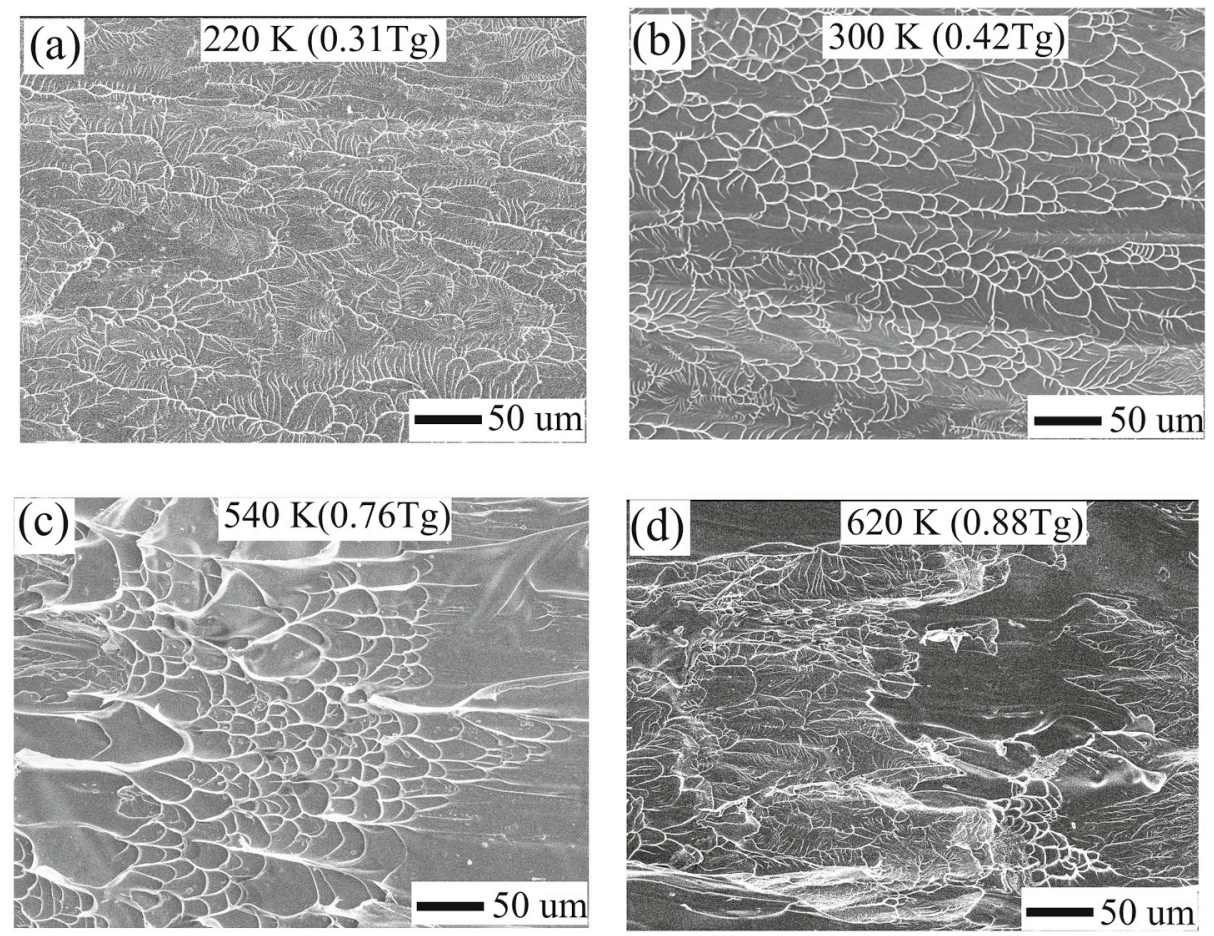

Fig. 4. Shear fracture surface morphologies for Zr-based MG at different temperatures. (a) $220 \mathrm{~K}\left(0.31 \mathrm{~T}_{\mathrm{g}}\right)$; (b) $300 \mathrm{~K}\left(0.42 \mathrm{~T}_{\mathrm{g}}\right)$; (c) $540 \mathrm{~K}\left(0.76 \mathrm{~T}_{\mathrm{g}}\right.$ ); (d) $620 \mathrm{~K}$ $\left(0.88 \mathrm{~T}_{\mathrm{g}}\right)$. 
(a)

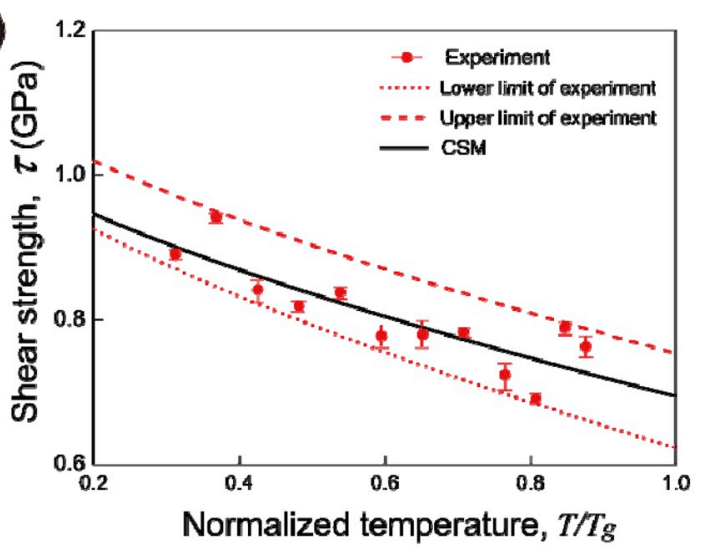

(b)

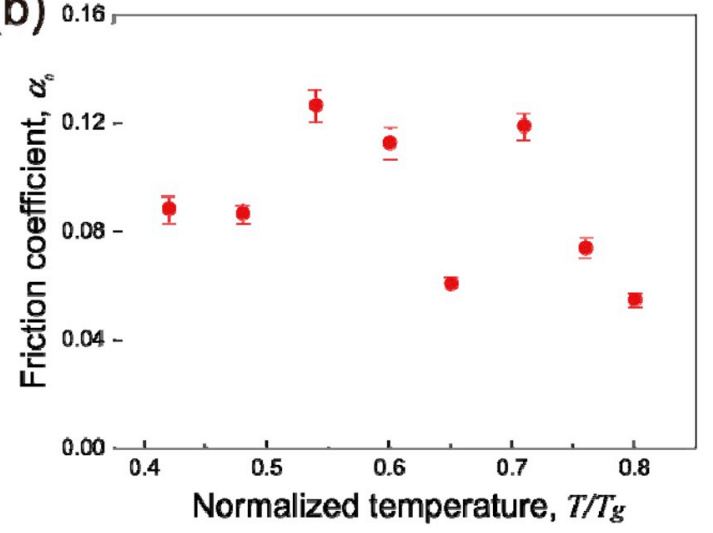

Fig. 5. (a) Comparing of shear strengths for Zr-based MG between experimental data and calculation results of CSM at different temperatures. (b) Friction coefficient of $\mathrm{M}-\mathrm{C}$ criterion at different temperatures.

be calculated according to Eq. (2). By this way, we calculated the value of $\alpha_{n}$ at different temperatures from $300 \mathrm{~K}$ to $570 \mathrm{~K}$, as listed in Table 1. As can be seen, the values of $\alpha_{n}$ are in the range of $0.06-0.13$, which are consistent with those reported by previous studies [35]. In contrast to the monolithic decrease of the shear strength (see Fig. 5b), $\alpha_{n}$ seems to have the high value $(>0.1)$ over the intermediate temperature range (from $380 \mathrm{~K}$ to $500 \mathrm{~K}$ ), while the low values of $\alpha_{n}$ appears at low temperatures $(\leq 340 \mathrm{~K})$ or high temperatures $(\geq 500 \mathrm{~K})$. As shown in Table 1 , the friction coefficient suddenly drops at $0.65 \mathrm{Tg}$, which may be caused by the transition from the inhomogeneous deformation (shear banding) to homogeneous deformation at about $0.65 \mathrm{Tg}$. The transition results in an anomaly of the shear strength at this point. Based on the $\mathrm{M}-\mathrm{C}$ yield criterion, the value of friction coefficient is dependent on the shear strength. Hence, the friction coefficient suddenly drops due to the transition of deformation mode. Moreover, the value of friction coefficient indicates that the normal stress effect changes with temperature. For MGs, the normal stress effect is closely related to the shear induced dilation at the atomic scale [33]. Hence, the friction coefficient also reveals that the shear induced dilation has different effect on the fracture behavior with temperature changing.

\section{Conclusions}

In summary, we designed a unique Anti-FPB test method to measure the pure shear strength of MGs. The shear strength in a Zr-based MG has been obtained by the anti-FPB shear test method over a wide range temperature from $220 \mathrm{~K}\left(0.31 T_{\mathrm{g}}\right)$ to $620 \mathrm{~K}\left(0.88 T_{\mathrm{g}}\right)$. The experimental result confirms that the shear strength is less than the half of compressive strength due to the normal stress effect. These strength data are used to verify the CSM and the M - C yield criterion, from which some key parameters in these models are determined. The measured pure shear strengths over different temperatures are important for constructing the yield criterion and understanding the shear dilation nature in MGs.

\section{Declaration of competing interest}

The authors declares no conflict of interest on the work.

\section{CRediT authorship contribution statement}

Cen Chen: Resources, Methodology, Investigation, Data curation, Writing - original draft. Baoan Sun: Conceptualization, Writing - review \& editing. Wei Hua Wang: Supervision, Conceptualization. Tzu Chiang Wang: Methodology, Supervision.

\section{Acknowledgements}

This work was supported by the National Natural Science Foundation of China [Grant Nos.11702295, 11790292, 11602272, 11602270, 11790291] and the Strategic Priority Research Program of the Chinese Academy of Sciences [Grant No. XDB22040503 and XDPB0601], the National Key Research and Development Plan [Grant No. 2016YFB0300501 \& 2017YFB0903902], and the Key Research Program of Frontier Sciences [QYZDY-SSW-JSC017]. B. A. Sun thanks the support from NSFC with the Grant No. 51822107 and 51671121, National Key Research and Development Plan (Grant No. 2018YFA0703603) and the Natural Science Foundation of Guangdong Province (Grant No. 2019B030302010).

\section{Appendix A. Supplementary data}

Supplementary data to this article can be found online at https://doi. org/10.1016/j.intermet.2019.106690.

\section{References}

[1] C.A. Schuh, A.C. Lund, Atomistic basis for the plastic yield criterion of metallic glass, Nat. Mater. 2 (2003) 449-452.

[2] L.Y. Chen, Z.D. Fu, G.Q. Zhang, X.P. Hao, Q.K. Jiang, X.D. Wang, Q.P. Cao, H. Franz, Y.G. Liu, H.S. Xie, New class of plastic bulk metallic glass, Phys. Rev. Lett. 100 (2008), 075501.

[3] R.D. Conner, W.L. Johnson, N.E. Paton, W.D. Nix, Shear bands and cracking of metallic glass plates in bending, J. Appl. Phys. 94 (2003) 904-911.

[4] R.D. Conner, Y. Li, W.D. Nix, W.L. Johnson, Shear band spacing under bending of Zr-based metallic glass plates, Acta Mater. 52 (2004) 2429-2434.

[5] J.J. Lewandowski, A.L. Greer, Temperature rise at shear bands in metallic glasses, Nat. Mater. 5 (2006) 15-18.

[6] C.A. Schuh, T.C. Hufnagel, U. Ramamurty, Mechanical behavior of amorphous alloys, Acta Mater. 55 (2007) 4067-4109.

[7] B.A. Sun, W.H. Wang, The fracture of bulk metallic glasses, Prog. Mater. Sci. 74 (2015) 211-307.

[8] W.H. Wang, The elastic properties, elastic models and elastic perspectives of metallic glasses, Prog. Mater. Sci. 57 (2012), 487-656.

[9] M.H. Cohen, D. Turnbull, Molecular transport in liquids and glasses, J. Chem. Phys. 31 (1959) 1164-1169.

[10] A.S. Argon, Plastic deformation in metallic glasses, Acta Metall. 27 (1979) 47-58.

[11] W.L. Johnson, K. Samwer, A universal criterion for plastic yielding of metallic glasses with a (T/Tg) ${ }^{2 / 3}$ temperature dependence, Phys. Rev. Lett. 95 (2005) 195501.

[12] M.D. Demetriou, J.S. Harmon, M. Tao, G. Duan, K. Samwer, W.L. Johnson, Cooperative shear model for the rheology of glass-forming metallic liquids, Phys. Rev. Lett. 97 (2006), 065502.

[13] Z.F. Zhang, J. Eckert, L. Schultz, Difference in compressive and tensile fracture mechanisms of $\mathrm{Zr}_{59} \mathrm{Cu}_{20} \mathrm{Al}_{10} \mathrm{Ni}_{8} \mathrm{Ti}_{3}$ bulk metallic glass, Acta Mater. 51 (2003) 1167-1179.

[14] R.T. Qu, Z.F. Zhang, A universal fracture criterion for high-strength materials, Sci. Rep. 3 (2013), 570-570.

[15] J. Caris, J.J. Lewandowski, Pressure effects on metallic glasses, Acta Mater. 58 (2010) 1026-1036. 
[16] F. Faupel, P.W. Hüppe, K. Rätzke, Pressure dependence and isotope effect of selfdiffusion in a metallic glass, Phys. Rev. Lett. 65 (1990) 1219.

[17] L. Anand, C. Su, A theory for amorphous viscoplastic materials undergoing finite deformations, with application to metallic glasses, J. Mech. Phys. Solids 53 (2005) $1362-1396$.

[18] Y.Q. Cheng, E. Ma, Intrinsic shear strength of metallic glass, Acta Mater. 59 (2011) 1800-1807.

[19] A.S. Khan, A. Pandey, T. Stoughton, Evolution of subsequent yield surfaces and elastic constants with finite plastic deformation. Part II: a very high work hardening aluminum alloy (annealed 1100 Al), Int. J. Plast. 26 (2010) 1421-1431.

[20] A.S. Khan, R. Kazmi, A. Pandey, T. Stoughton, Evolution of subsequent yield surfaces and elastic constants with finite plastic deformation. Part-I: a very low work hardening aluminum alloy (Al6061-J6511), Int. J. Plast. 25 (2009) 1611-1625.

[21] H.A. Bruck, T. Christman, A.J. Rosakis, W.L. Johnson, Quasi-static constitutive behavior of $\mathrm{Zr}_{41.25} \mathrm{Ti}_{13.75} \mathrm{Ni}_{10} \mathrm{Cu}_{12.5} \mathrm{Be}_{22.5}$ bulk amorphous alloys, Scripta. Metall. Et. Mater. 30 (1994) 429-434.

[22] K.M. Flores, R.H. Dauskardt, Mode II fracture behavior of a Zr-based bulk metallic glass, J. Mech. Phys. Solids 54 (2006) 2418-2435.

[23] D.E. Walrath, D.F. Adams, The Iosipescu shear test as applied to composite materials, Exp. Mech. 23 (1983) 105-110.

[24] J. Morton, H. Ho, M.Y. Tsai, G.L. Farley, An evaluation of the Iosipescu specimen for composite materials shear property measurement, J. Compos. Mater. 26 (1992) $708-750$.

[25] C. Chen, M. Gao, C. Wang, W.H. Wang, T.C. Wang, Fracture behaviors under pure shear loading in bulk metallic glasses, Sci. Rep. 6 (2016) 39522.
[26] N. losipescu, New accurate method for single shear testing of metals, J. Mater. 2 (1967) 537-566.

[27] J.L. Sullivan, B.G. Kao, H.V. Oene, Shear properties and a stress analysis obtained from vinyl-ester losipescu specimens, Exp. Mech. 24 (1984) 223-232.

[28] X.K. Xi, D.Q. Zhao, M.X. Pan, W.H. Wang, Y. Wu, J.J. Lewandowski, Fracture of brittle metallic glasses: brittleness or plasticity, Phys. Rev. Lett. 94 (2005) 125510.

[29] Y.H. Liu, C.T. Liu, W.H. Wang, A. Inoue, T. Sakurai, M.W. Chen, Thermodynamic origins of shear band formation and the universal scaling law of metallic glass strength, Phys. Rev. Lett. 103 (2009), 065504.

[30] X.F. Cao, M. Gao, L.Z. Zhao, W.H. Wang, H.Y. Bai, Microstructural heterogeneity perspective on the yield strength of metallic glasses, J. Appl. Phys. 119 (2016) 605-609.

[31] R.T. Qu, J. Eckert, Z.F. Zhang, Tensile fracture criterion of metallic glass, J. Appl. Phys. 109 (2011), 083544-083544.

[32] K.M. Flores, R.H. Dauskardt, Mean stress effects on flow localization and failure in a bulk metallic glass, Acta Mater. 49 (2001) 2527-2537.

[33] M.Q. Jiang, G. Wilde, J.H. Chen, C.B. Qu, S.Y. Fu, F. Jiang, L.H. Dai, Cryogenictemperature-induced transition from shear to dilatational failure in metallic glasses, Acta Mater. 77 (2014) 248-257.

[34] M. Bletry, P. Guyot, J.J. Blandin, J.L. Soubeyroux, Free volume model: hightemperature deformation of a Zr-based bulk metallic glass, Acta Mater. 54 (2006) 1257-1263.

[35] A.C. Lund, C.A. Schuh, The Mohr-Coulomb criterion from unit shear processes in metallic glass, Intermetallics 12 (2004) 1159-1165. 